\title{
Innovative Physics and Engineering Research in Nuclear Medicine and Molecular Imaging: A Message from the Associate Editor
}

\author{
Jae Sung Lee ${ }^{1}$
}

Received: 28 September 2015 / Revised: 13 October 2015 / Accepted: 14 October 2015 / Published online: 26 October 2015

(C) Korean Society of Nuclear Medicine 2015

New imaging instruments and analysis techniques have led to major advances in nuclear medicine and molecular imaging. The invention of the Anger scintillation camera began the modern era of nuclear medicine imaging, and the camera is still widely used today [1]. Positron emission tomography (PET) and single photon emission computed tomography (SPECT), based on tomographic image reconstruction algorithms, offer much higher detection power and localization accuracy than conventional scintigraphy. The introduction of dual-modality PET/computed tomography (CT) and SPECT/ CT systems in the late 1990s was a revolutionary advance in modern diagnostic imaging [2, 3].

During the past decade, we witnessed many remarkable technical advances in nuclear medicine and related hybrid imaging systems. The advances in scintillation crystals, photosensors, and electronics have led to the wide use of time-of-flight (TOF) measurement in PET, which yields improved image quality or reduces scan time or radiation dose [4]. Continuing efforts to further improve TOF measurement techniques would yield additional success stories for this technology, e.g., accurate simultaneous estimation of radioactivity and attenuation without the use of transmission data and sparse PET with an extremely long axial field-of-view for performing single-bed whole-body PET studies. There have also been advances in simultaneous PET/magnetic resonance imaging (MRI) technology, which has been regarded as one of

Jae Sung Lee

jaes@snu.ac.kr

1 Departments of Nuclear Medicine and Biomedical Sciences, Seoul National University College of Medicine, Seoul 110-744, South Korea most difficult technical challenges in biomedical engineering; however, it promises several advantages over PET/CT, including a smaller radiation dose, better soft tissue contrast, and fewer motion artifacts [5]. The key advance to overcome this challenge was a semiconductor photosensor that is insensitive to static and time-varying magnetic fields. Semiconductor photosensors are continuously evolving and eventually likely to replace the conventional photomultiplier tubes (PMTs) in clinical PET/CT and PET/MRI. PET detectors and systems based on silicon photomultiplier (SiPM), which is a semiconductor photosensor with sufficiently high internal gain, have already outperformed the PMT-based detectors and systems [6].

Research on improving nuclear medicine and hybrid imaging instruments will continue, and some of the findings will lead to the design of new products. The development of lowcost imaging devices that do not compromise diagnostic performance is another area of increasing interest. In addition, new algorithms and software techniques that make nuclear medicine imaging more accurate and quantitative and make the therapeutic effects of nuclear medicine more predictable are highly important. Nuclear Medicine and Molecular Imaging is well positioned to participate in reporting on the important results of these scientific investigations and new technological developments because the journal is expanding its scope to include nuclear medicine physics, radiochemistry/ pharmacy, and molecular imaging [7]. As a starting point for this expansion, we have invited renowned scientists to contribute review papers that cover various important issues in the field of nuclear medicine physics and engineering. I express my sincere gratitude to the authors, who will contribute review papers in the following issues, and look forward to receiving future articles that will inform our readers of the latest investigations on imaging instruments, image generation, and analysis techniques. Finally, I am pleased to serve as the Associate 
Editor of Nuclear Medicine and Molecular Imaging and to have the opportunity to contribute this editorial.

Conflict of Interest Jae Sung Lee declares no conflict of interest.

\section{References}

1. Anger HO. Scintillation camera. Rev Sci Instrum. 1957;29:27-33.
2. Seo Y, Mari C, Hasegawa BH. Technological development and advances in single-photon emission computed tomography/computed tomography. Semin Nucl Med. 2008;38:177-98.

3. Townsend DW. Dual-modality imaging: combining anatomy and function. J Nucl Med. 2008;49:938-55.

4. Conti M. Focus on time-of-flight PET: the benefits of improved time resolution. Eur J Nucl Med Mol Imaging. 2011;38:1147-57.

5. Vandenberghe S, Marsden PK. PET-MRI: a review of challenges and solutions in the development of integrated multimodality imaging. Phys Med Biol. 2015;60:R115-54.

6. Roncali E, Cherry SR. Application of silicon photomultipliers to positron emission tomography. Ann Biomed Eng. 2011;39:1358-77.

7. Lee DS. Message from the new Editor-in-Chief. Nucl Med Mol Imaging. 2015;49:1-2. doi:10.1007/s13139-015-0325-5. 